\section{Quantum Dots and Nanowires Demonstrate Potential for Efficient Solar Cells}

As solar power technology becomes more widely adopted, intensive research efforts are underway to find the next generation of inexpensive but efficient photovoltaics beyond crystalline silicon. One example is quantum-dot-sensitized solar cells, which harvest sunlight using quantum dots adsorbed on a network of $\mathrm{TiO}_{2}$ nanoparticles. However, the disordered structure of these mesoscopic $\mathrm{TiO}_{2}$ networks can allow photogenerated electrons to recombine with holes, which reduces overall efficiency. K. Leschkies and colleagues at the University of Minnesota have combined quantum dots (QDs) with ordered arrays of single-crystal semiconductor nanowires to produce photovoltaic devices in which electron-hole recombination is significantly reduced.

As reported in the June issue of Nano Letters (p. 1793; DOI: 10.1021/nl070430o), the research group theorized that the ordered nanowire array would more efficiently guide photogenerated electrons to the photoanode than disordered $\mathrm{TiO}_{2}$ networks. To fabricate the device, the researchers began by growing $\mathrm{ZnO}$ nanowires onto a transparent, conducting $\mathrm{SnO}_{2}$ substrate. The wires had lengths between $2 \mu \mathrm{m}$ and $12 \mu \mathrm{m}$ and diameters of 75-125 $\mathrm{nm}$. Separately, the group prepared CdSe nanocrystals approximately $3 \mathrm{~nm}$ in diameter, which they capped with mercaptopropionic acid (MPA). By immersing the nanowires in a methanol dispersion of the QDs, the researchers were able to attach the QDs to the nanowires. Treating the nanowires with oxygen plasma before immersion significantly increased the attachment efficiency. To complete the device fabrication, the group assembled the QD-decorated nanowire photoanode face-to-face with a $\mathrm{SnO}_{2}$ photocathode, filling the $25-\mu \mathrm{m}$ space between the electrodes with a hybrid liquid electrolyte.

The resulting devices displayed a photovoltaic effect and clear evidence that photogenerated electrons in the QDs were injected into the nanowires. While the overall energy-conversion efficiency of the system was less than $0.5 \%$, this was limited primarily by the surface area of the nanowires. The internal quantum efficiencies were as high as $58 \%$, indicating that the nanowires efficiently collected and guided photogenerated electrons. The researchers reported that their solar cells were stable for a few hours to a few days in air. They said that liquid electrolyte is known to degrade quantum dots over time, and that optimization of the cells will require a transition to a different elec- trolyte, which will be the subject of future work. Given these results, devices based on single-crystal nanowires sensitized with solar-tailored quantum dots may someday become the next-generation photovoltaic system of choice.

Colin McCormick

\section{0-nm Wide Laser Tunability Range Obtained in Tm:LiLuF 4 Crystal}

Solid-state lasers based on thulium and emitting at around $1.9 \mu \mathrm{m}$ are interesting as they can be continuously tuned in wavelength from $1.8 \mu \mathrm{m}$ to $2 \mu \mathrm{m}$. They provide an appropriate tool for highresolution molecular spectroscopy, atmospheric remote sensing, and medical surgery, as several absorption lines of chemical compounds, such as $\mathrm{H}_{2} \mathrm{O}, \mathrm{CO}_{2}$, and $\mathrm{NO}_{2}$, are present in this range. Tmdoped active media can be pumped by commercially available high-power InGaAs laser diodes at wavelengths around $790 \mathrm{~nm}$, allowing the implementation of efficient, compact, and rugged laser sources.

N. Coluccelli and co-workers from the Politecnico di Milano and the Istituto di Fotonica e Nanotecnologie-CNR in Milan, Italy, and F. Cornacchia and co-workers from the Pisa University and National Enterprise for Nano Science and Technology-INFM Pisa, Italy, have reported in the July issue of Optics Letters (p. 2040) roomtemperature continuous-wave laser action in Tm:LiLuF 4 crystals with a tunability range of $230 \mathrm{~nm}$, an output power of 1.15 $\mathrm{W}$, and a slope efficiency with respect to the incident power up to $46 \%$, which represents, the researchers said, the highest value ever demonstrated with diodepumped Tm-doped fluoride crystals.

The researchers grew crystals of Tm:LiLuF 4 with scheelite structure (with two axes of equal length $a$, and a third of length $c$ ) from the melt by the Czochralski technique at $860^{\circ} \mathrm{C}$ in a highpurity $(99.999 \%)$ argon atmosphere from $\mathrm{LiF}$ and $\mathrm{LuF}_{3}$ powders and adding $8 \%$, $12 \%$, and $16 \%$ mol of $\mathrm{TmF}_{3}$ powder to the melt. $\mathrm{Tm}^{3+}$ substituted $\mathrm{Lu}^{3+}$ in the structure with a segregation coefficient close to one. The room-temperature absorption spectrum of $\mathrm{Tm}^{3+}$ in these crystals in the $800 \mathrm{~nm}$ region shows the most intense peak at $779.8 \mathrm{~nm}(\mathrm{FWHM}=7.6 \mathrm{~nm})$ for the polarization of the light parallel to the $c$-axis $(E \| c)$, a peak particularly suitable for diode pumping. The researchers found the maximum values for the emission cross section at $1911 \mathrm{~nm}$ for the $E \| a$ polarization $\left(0.4 \times 10^{-20} \mathrm{~cm}^{2}\right)$ and at 1886 $\mathrm{nm}$ for the $E \| c$ polarization $\left(0.31 \times 10^{-20}\right.$ $\mathrm{cm}^{2}$ ). The researchers performed the laser experiments in a three-mirror folded cavity using a cw $\mathrm{AlGaAs}$ laser diode as the pump source with a linearly polarized emission tuned at $\sim 780 \mathrm{~nm}$ and a maximum output power of $3.2 \mathrm{~W}$ as the pump source. The sample was placed inside the resonator in such a way to take advantage of the absorption cross section obtained along the $E \| c$ polarization. It was oriented at Brewster's angle to minimize Fresnel losses of both the pump and laser beams originating from the air- crystal interfaces. A $12 \%$ Tm doping level provided the highest output power of $1.15 \mathrm{~W}$ (at $3.2 \mathrm{~W}$ pump power) using a $5 \%$ output coupler. With a $1 \%$ output coupler, the slope efficiency slightly increased from $43 \%$ to $46 \%$, although the maximum output power was only of $1.05 \mathrm{~W}$ at $3.2 \mathrm{~W}$ pump power. By inserting a 2-mm thick birefringent quartz plate, oriented also at Brewster angle along the longest arm of the resonator, the researchers investigated the emission tuning range of $\mathrm{Tm}: \mathrm{LiLuF}_{4}$ crystals, obtaining the widest tuning range in a $12 \% \mathrm{Tm}_{\text {-doped }} \mathrm{LiLuF}_{4}$ crystal, from $1826 \mathrm{~nm}$ to $2056 \mathrm{~nm}$.

JOAN J. CARVAJAL

\section{Effects of Hydration on Electronic Properties of $\mathbf{C}_{60}$ Fullerene Accurately Calculated from First Principles}

The optical efficiency of semiconducting nanoparticles has triggered industrial interest for their incorporation into sensing and other electronics applications. Fullerenes, for example, which have been shown to increase the biostability of electrochemical sensors, are under investigation as Raman-active nanosensors and cytotoxic agents, and are considered one of the most versatile biologically important nanoparticles. However, biological applications typically include an aqueous environment. Consequently, an understanding of the interactions between nanoparticles and water and their effect on optical properties is paramount. Recently, R. Rivelino and F. de Brito Mota from Instituto de Fisica, Universidade Federal da Bahia, Brazil, used a sequential Monte Carlo/density functional theory (MC/DFT) scheme to examine the effects of hydration and the dielectric screening of water on the $C_{60}$ bandgap and density of states under ambient conditions.

As described in the June issue of Nano Letters (p. 1526; DOI: 10.1021/n1070308p), Rivelino and de Brito Mota used an atomistic model consisting of a periodic cell with one $\mathrm{C}_{60}$ molecule solvated by 1000 water molecules at standard temperature and pressure. The dispersion interaction 